\title{
Immunoblot Analysis of Horseradish Peroxidase Conjugates of Wheat Germ Agglutinin Before and After Retrograde Transport in the Rat Peripheral Nervous System ${ }^{1}$
}

\author{
M. L. SCHMIDT ${ }^{2}$ AND J. Q. TROJANOWSKI
}

The Division of Neuropathology, Department of Pathology and Laboratory Medicine, The University of Pennsy/vania School of Medicine, Philadelphia, Pennsylvania 19104

\begin{abstract}
Immunoblot studies were performed on tissue extracts of rats injected with horseradish peroxidase (HRP) conjugates of wheat germ agglutinin (WGA) using anti-HRP and antiWGA antisera. Immunoblots of uninjected, purified conjugates (WGA-HRP) demonstrated a 62-kilodalton (kd) conjugate (monomeric WGA conjugated to HRP). Other prominent immunobands included HRP (40 kd), monomeric WGA (22 kd), and 15- to 30-kd HRP breakdown products. Following injections of WGA-HRP into the submandibular gland of rats which survived $16 \mathrm{hr}$ to 8 days, immunoblots were performed on homogenates of injected submandibular glands and of superior cervical ganglia containing neurons retrogradely labeled with WGA-HRP. The anti-HRP antiserum detected unconjugated, 40-kd HRP and a 62-kd immunoband corresponding to WGA-HRP in the superior cervical ganglia and submandibular glands. No immunobands were detected with the antiserum to WGA in superior cervical ganglia homogenates. Blots of submandibular gland homogenates harvested $24 \mathrm{hr}$ after a WGA-HRP injection, but not at later time points, contained an immunoband reactive with the anti-WGA antiserum; it corresponded to monomeric WGA.

These studies analyze for the first time the molecular composition of WGA-HRP conjugates before and after retrograde transport; they provide a novel approach for probing the intraneuronal transport and degradation of proteins. We conclude that morphologically defined endocytic pathways using protein markers reflect the endocytosis and transport of immunochemically altered and unaltered forms of the markers.
\end{abstract}

The introduction into the neurobiology of native and derivatized ligands has permitted the delineation of novel pathways for the intraneuronal transport of proteins internalized by adsorptive endocytosis (Schwab et al., 1979; Harper et al., 1980; Margolis et al., 1981; Trojanowski et al., 1981; LaVail et al., 1983; Trojanowski and

Received January 16, 1985; Revised March 18, 1985

Accepted March 18, 1985

\footnotetext{
1 This work was supported by National Institutes of Health Grants NS16723 and Teacher investigator Development Award KO7 NS-00762 (to J. Q. T.) from the National Institute of Neurological and Communicative Disorders and Stroke, and Grant F32 EY-05568 (to M. L. S.) from the National Eye Institute. We thank Ms. M. Obrocka for expert technical assistance and Dr. V. M. $-Y$. Lee for advice on the immunoblots.

${ }^{2}$ To whom correspondence should be addressed.
}

Gonatas, 1983; Broadwell et al., 1984; LaVail and Sugino, 1984). The demonstration of the interneuronal transfer of some ligands following intraneuronal transport, such as occurs with the wheat germ agglutinin (WGA) and cholera toxin (Schwab et al., 1979; Gerfen et al., 1982; Itaya and Van Hoesen, 1982; Ruda and Coulter, 1982; LaVail et al., 1983; Trojanowski and Schmidt, 1984) provides new opportunities to study the mechanisms underlying this poorly understood phenomenon (Grafstein and Forman, 1980).

Most morphological studies of the in vivo internalization and transport of lectins such as WGA utilize a derivatized form of the ligand (Schwab ct al., 1978, 1979; Gonatas et al., 1979; Gonatas, 1982; Trojanowski, 1983a). For example, WGA, covalently conjugated to horseradish peroxidase (HRP), yields a hybrid probe (WGAHRP) which can be histochemically visualized due to the peroxidatic activity of HRP. This hybrid probe has properties in vivo which clearly distinguish it from native HRP (Gonatas et al., 1979; Gonatas, 1982; Trojanowski, 1983a). Such data support the assumption that the peroxidatic activity visualized in tissue sections corresponds to the location of the lectin hybrid. This assumption has not been subjected to detailed scrutiny, although unconjugated HRP is present in WGAHRP preparations (Trojanowski, 1983b). Thus, in morphological studies which employ WGA-HRP, the histochemically visualized peroxidatic activity could be due to both unconjugated and conjugated HRP. Ambiguity on this point may limit conclusions drawn from studies based on the use of WGA-HRP. To address this issue, we analyzed a WGA.HRP preparation in immunoblots before and after injections of this probe into the submandibular gland of rats and following retrograde transport of the lectin hybrid to neurons of superior cervical ganglia.

\section{Materials and Methods}

Chemicals and immunochemicals. WGA-HRP (lot 44F-8120), WGA (lot 33F-9605), HRP (lot 23F-8580), $N$-acetylglucosamine, and rabbit anti-WGA and rabbit anti-HRP antisera were purchased from Sigma Chemical Co. This WGA-HRP preparation was produced using a heterobifunctional reagent (O'Sullivan et al., 1978) and purified after conjugation by affinity chromatography (Sigma). HRP-labeled goat anti-rabbit IgG was purchased from Cappel Laboratories. Low molecular weight markers were purchased from Bio-Rad Laboratories.

Experimental animal procedures. All submandibular gland injections of WGA-HRP or phosphate-buffered saline (PBS) were performed on Chloropent (0.3 $\mathrm{ml} / 100 \mathrm{gm}$ )-anesthetized, 150 - to 300 -gm male Sprague Dawley rats. For the immunochemical studies, three rats per time interval received bilateral injections of $1 \mathrm{mg}$ of WGA-HRP in $10 \mu$ l of PBS in each submandibular gland except for the 16- and 36-hr groups which included two rats. Two groups of controls (2 and 3 rats/group) received bilateral submandibular gland injections of $10 \mu$ l of PBS and survived 2 or 5 days.

For the histochemical experiments, $50 \mu \mathrm{g}$ of WGA-HRP in $10 \mu \mathrm{l}$ of PBS were injected into both submandibular glands of one rat per survival interval 
of 6,9 , and 12 days. Another rat received an injection of $1 \mathrm{mg}$ of WGA-HRP in $10 \mu \mathrm{l}$ of PBS, and a second rat was similarly injected with $10 \mu \mathrm{l}$ of PBS alone.

Preparation of tissues for immunochemistry. After survival times of 16 , 24 , and $36 \mathrm{hr}$, as well as 3,5 , and 8 days, the WGA-HRP-injected rats used for the immunoblot experiments were anaesthetized in ether and perfused with $500 \mathrm{ml}$ of PBS through the left ventricle. For each time point, except the 16- and 36 -hr intervals, six superior cervical ganglia were obtained as well as a portion of a submandibular gland from one rat in each group. For the 16- and 36-hr survival times, four superior cervical ganglia and a portion of the submandibular gland from one 16- and one 36-hr rat were used. These tissues were then analyzed in immunoblots as previously described (Schmidt et al., 1985).

Briefly, pooled superior cervical ganglia from each group of rats and a portion of a submandibular gland ( 40 to $110 \mathrm{mg}$ ) from one rat in each group were separately homogenized in a 3-fold volume $(\mathrm{w} / \mathrm{v})$ of $5 \mathrm{~mm}$ Tris- $\mathrm{HCl}$ buffer $(\mathrm{pH} 7.6)$ containing $0.1 \mathrm{M}$ tosyl-1-arginine-methyl-ester-hydrochloride with $0.1 \mathrm{~mm}$ EDTA, $0.1 \mathrm{~mm}$ EGTA, and $0.1 \%$ sodium dodecyl sulfate. The two groups of PBS-injected control rats were similarly treated.

To evaluate the effect of the superior cervical ganglion and submandibular gland proteins on the electrophoretic mobility of WGA-HRP and on the specificity of the antisera to HRP or WGA in immunoblots, six superior cervical ganglia and one submandibular gland from each of two additional groups of uninjected rats (3 rats/group) were homogenized in the same manner. To $100 \mu \mathrm{l}$ of the superior cervical ganglion homogenate were added either $10 \mu \mathrm{g}$ of WGA or $10 \mu \mathrm{g}$ of WGA-HRP prior to immunochemical analysis. To separate $100-\mu$ l aliquots of the submandibular gland homogenate were added 10,50 , and $100 \mu \mathrm{g}$ of either WGA or WGA-HRP prior to immunochemical study.

Prior to SDS-PAGE, the samples were frozen and thawed five times, heated to $100^{\circ} \mathrm{C}$ for $2 \mathrm{~min}$, and centrifuged for $30 \mathrm{~min}$ at $20,000 \times \mathrm{g}$. The supernatants were mixed with equal amounts of Laemmli sample buffer (Laemmli, 1970), heated for $2 \mathrm{~min}$ to $100^{\circ} \mathrm{C}$, microfuged, and then subjected to SDS-PAGE.

To determine whether any immunoreactive protein remained in the initial pellet, the pellets obtained from superior cervical ganglia and submandibular glands of the 36-hr WGA-HRP-injected rats following the first homogenization step were separately dissolved in $100 \mu \mathrm{l}$ of sample buffer and examined in immunoblots.

SDS-PAGE and immunoblot procedures. The samples were subjected to discontinuous SDS-PAGE in gradient gels. For comparison, the electrophoretic mobility of uninjected WGA and WGA-HRP was evaluated in $10 \%$ or $15 \%$ gels stained with Coomassie blue. The polyacrylamide content of all of the gradient gels used here was 7.5 to $15 \%$. The gels were $0.75 \mathrm{~mm}$ thick, and combs with 2-cm-wide wells were used for all samples containing tissue homogenates. WGA, HRP, and WGA-HRP dissolved in Laemmli sample buffer but unmixed with tissue homogenates were also subjected to SDSPAGE using similar gradient gels and combs with $3.5-$ or $0.5-\mathrm{cm}$-wide wells. Low molecular weight markers were run in parallel with the samples.

Proteins in the gels were transferred to nitrocellulose paper according to a modification (Lee et al., 1984; Schmidt et al., 1985) of the procedure of Towbin et al. (1979). The nitrocellulose replicas of the gels were cut into strips and blocked for 1 to $16 \mathrm{hr}$ in PBS containing $1 \%$ bovine serum albumin (PBS-BSA) followed by incubation in antiserum for $16 \mathrm{hr}$ to 3 days. The antiWGA and anti-HRP antisera were applied to the strips diluted in PBS-BSA in a range of dilutions from 1:100 to 1:2000. After washing in PBS with $0.5 \%$ Tween, peroxidase-labeled goat anti-rabbit lgG diluted 1:1000 in PBS-BSA was applied for $2 \mathrm{hr}$. Finally, the strips were washed in PBS with Tween and reacted in 4-chloro-1-naphthol with hydrogen peroxide. Under the conditions described here, the HRP in the replicas was devoid of enzymatic activity; the reaction product seen in the immunostained nitrocellulose strips was due to the antibodies bound to the replicas (Schmidt et al., 1985).

Immunoblot controls. A number of controls were performed to validate the results in the experimental animals. These included immunoblots of tissue samples from animals injected with PBS alone. Immunoblots were also performed on known amounts of WGA-HRP, WGA, and HRP electrophoresed alone or admixed with homogenates of submandibular glands or superior cervical ganglia from uninjected rats. In other control experiments, $2 \mu \mathrm{g}$ of WGA-HRP were incubated with $1 \mathrm{mg}$ of $\mathrm{N}$-acetylglucosamine at $4^{\circ} \mathrm{C}$ overnight and then subjected to immunochemical study.

Furthermore, the immunochemical stability of WGA-HRP solubilized in PBS was evaluated by keeping aliquots of this solution for up to 3 months at $4^{\circ} \mathrm{C}$ prior to immunoblot analysis. The optimal transfer time ( $30 \mathrm{~min})$ was determined by conducting transfers for variable lengths of time (15 to $90 \mathrm{~min}$ ) and evaluating the Coomassie blue-stained gels after transfer and the immunoblots prepared from these gels. Finally, the electrophoretic mobility of WGA, WGA-HRP, and HRP dissolved in sample buffer devoid of dithiothreitol was evaluated in immunoblots and in Coomassie blue-stained gels.

Analysis of WGA-HRP in nondenaturing gels. The electrophoretic mobility and peroxidatic activity of WGA-HRP was analyzed using non-denaturing gels stained with Coomasie Blue or incubated with 4-chloro-1-naphthol with hydrogen peroxide as described previously (Schmidt et al., 1985). The peroxidatic activity of HRP is retained in these gels.

Histochemical procedures. All of the rats used for histochemistry were lethally etherized and perfused, and frozen sections of the superior cervical ganglia were prepared for histochemical staining with the chromogen tetramethylbenzidine as previously described (Schmidt et al., 1985). Hematoxylin and eosin-stained paraffin sections of the submandibular glands from the rats that received $1 \cdot \mathrm{mg}$ injections of WGA-HRP or PBS alone were also examined.

\section{Results}

SDS-PAGE analysis of WGA-HRP. The electrophoretograms of uninjected WGA-HRP preparations in gradient gels revealed three prominent bands of $M_{r}=62,000,40,000$, and 22,000 (Fig. 1, $A$ and C). The same molecular weight species were seen when WGA-HRP was analyzed in $10 \%$ or $15 \%$ gels. The 40 - and 22 -kilodalton (kd) molecular weight bands co-migrated with HRP and monomeric WGA, respectively, in similar gels of these unconjugated proteins. The $62-k d$ band represents a 1:1 WGA-HRP conjugate (40-kd HRP plus 22-kd WGA). The conjugation of HRP to WGA with metamaleimidobenzoyl- $N$-hydroxysuccinimide ester, which was used to prepare the conjugates studied here (Sigma), favors low molecular weight conjugates (O'Sullivan et al., 1978). This is not true of all heterobifunctional cross-linkers (Nygren, 1982).

The molecular weight of WGA seen here is in the range $(16,000$ to 23,000) established for monomers of this lectin (Nagata and Burger, 1974; Rice and Etzler, 1975; Goldstein and Hayes, 1978; Margolis et al., 1981; Steindler and Bradely, 1983; Trojanowski
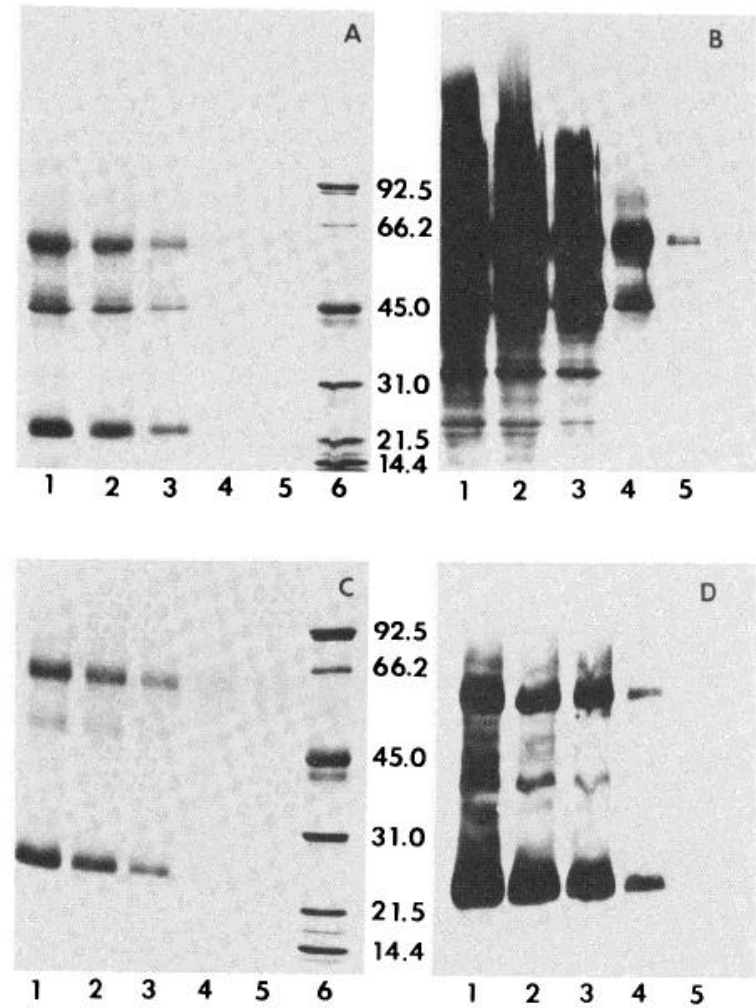

Figure 1. Analysis of different concentrations of WGA-HRP in lanes 1 to $5(15,10,5,1$, and $0.1 \mu \mathrm{g}$ of WGA-HRP/0.5-cm-wide lane, respectively) of gradient gels stained with Coomassie blue $(A$ and $C)$ or following transfer to nitrocellulose sheets and immunostaining with antiserum to either HRP diluted 1:1000 $(B)$ or WGA diluted 1:500 $(D)$. Low molecular weight markers in kilodaltons are present in lane 6 of the Coomasie blue-stained gels. 


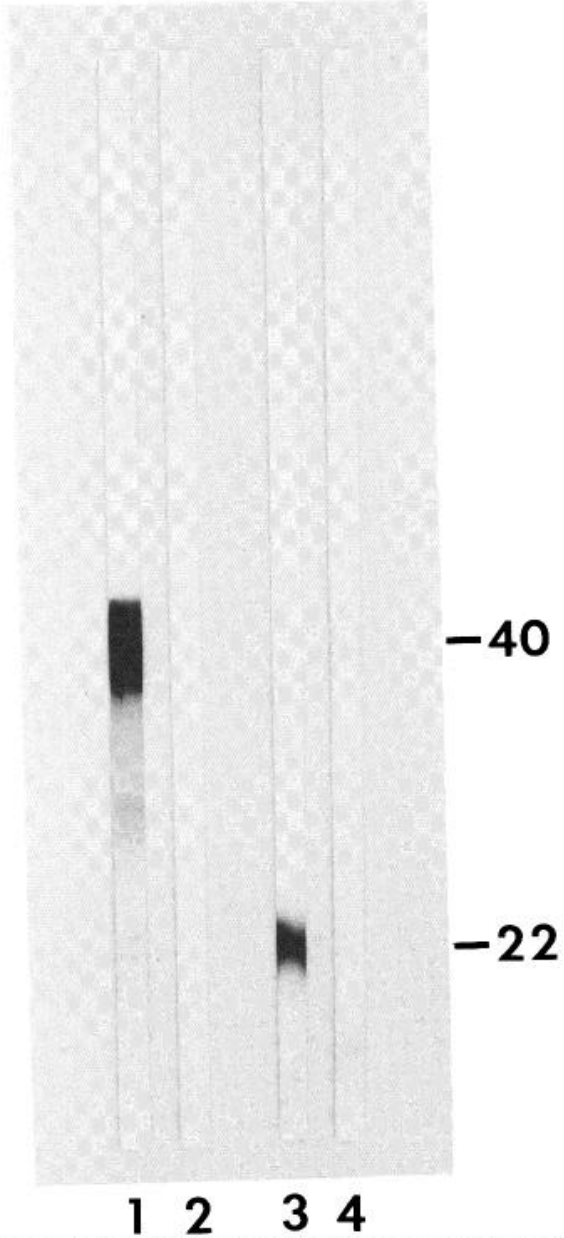

Figure 2. Strips from nitrocellulose replicas of gradient gels containing either HRP (strips 1 and 2) or WGA (strips 3 and 4). The strips were immunostained with antiserum to HRP (strips 1 and 4 ) or antiserum to WGA (strips 2 and 3). The antisera were applied to the strips in dilutions of 1:1000, and $4 \mu \mathrm{g}$ of HRP or WGA were loaded per $3.5-\mathrm{cm}$-wide lane. Molecular weights in kilodaltons are indicated on the right. 1983b; Raikhel et a., 1984). The variable electrophoretic mobility of WGA may be due to its unusual amino acid composition (Nagata and Burger, 1974; Raikhel et al., 1984). The electrophoretic mobility of WGA, WGA-HRP, or HRP did not change when dithiothreitol was omitted from the sample buffer.

Antisera specificity and immunochemistry of uninjected WGA$H R P$. The proteins in WGA-HRP preparations immunoreactive with antiserum to WGA or to HRP are seen in immunoblots (Fig. 1, $B$ and $C)$ prepared from gels run in parallel with those in Figure 1, $A$ and $C$. These immunoblots demonstrate that antibodies are more sensitive than Coomassie blue staining for the detection of proteins in WGA-HRP preparations. The immunoblot method detected proteins which were not seen in Coomassie blue-stained gels. For example, the antiserum to WGA detected a 36-kd immunoband in addition to the 22-kd (monomeric WGA) and 62-kd (WGA-HRP) bands seen in the Coomassie blue-stained gels. This $36-k d$ immunoband probably corresponds to WGA dimers or aggregates. The antiserum to HRP also demonstrated bands not seen in the Coomassie blue-stained gels. In addition to the free HRP (the broad 40- to 45-kd immunoband) and the 62-kd band (WGA-HRP), minor 15- to 30-kd bands were evident in immunoblot lanes containing 5 to $15 \mu \mathrm{g}$ of WGA-HRP. These immunobands correspond to spontaneous HRP breakdown products (Schmidt et al., 1985).

To verify the specificity of these two antisera, and to establish their sensitivity in immunoblots, we used them to stain adjacent strips from replicas of gels containing electrophoresed, uninjected WGA or HRP. Results from such an experiment are seen in Figure 2. These and other similar experiments confirmed that the anti-WGA antiserum did not cross-react with HRP and that the antiserum to HRP did not cross-react with WGA. The strips with HRP also contained spontaneous HRP breakdown products (Schmidt et al., 1985).

The specificity and sensitivity of these antisera were further established by varying the dirution of the anti-WGA and anti-HRP antisera applied to the replicas. Representative studies of this type are illustrated in Figure 3. Studies with the antiserum to WGA (Fig. 3, A, and $B$ ) demonstrate that WGA-HRP conjugates contain primarily two major bands recognized by this antiserum: a 22-kd band corresponding to monomeric WGA and a 62-kd band corresponding to a conjugate comprised of monomeric WGA and HRP. A weak immunoband migrating at approximately $36 \mathrm{kd}$ probably corre-

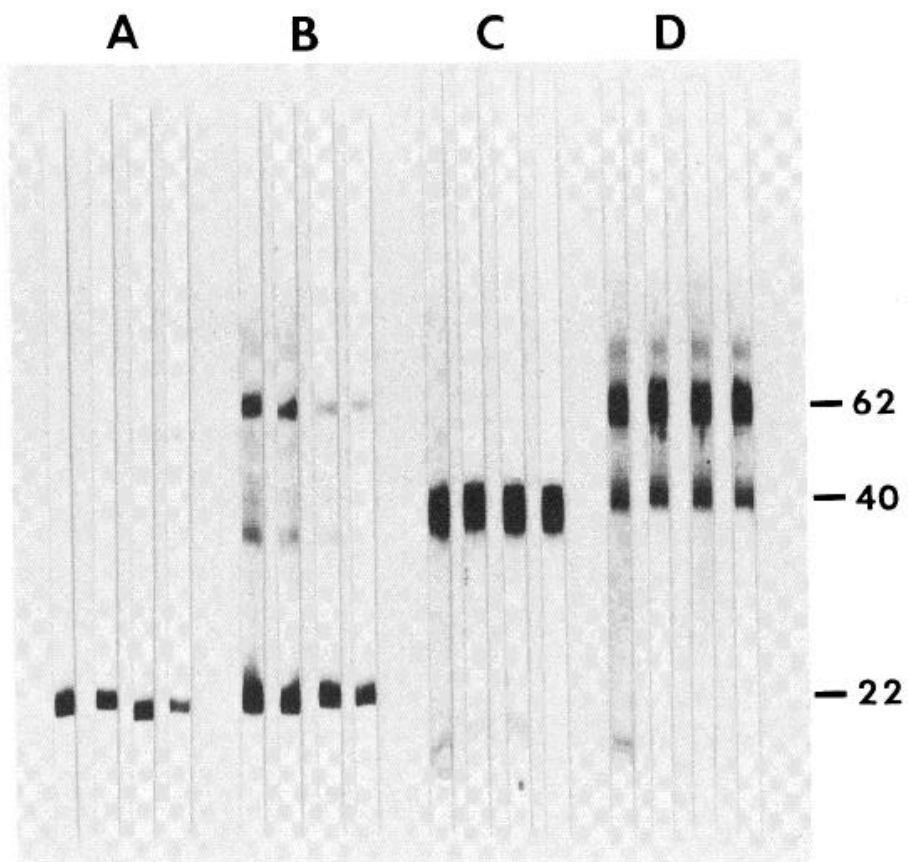

Figure 3. Strips from nitrocellulose replicas of gradient gels containing $2 \mu \mathrm{g}$ of WGA (group A), WGA-HRP (groups B and D), or HRP (group C) per $3.5-\mathrm{cm}$-wide lane. The strips were immunostained with different dilutions of antiserum to WGA ( $A$ and $B)$ or $\operatorname{HRP}(C$ and $D)$. The dilutions of each antiserum were 1:100 (strip 1 in each group), 1:250 (strip 2 in each group), 1:500 (strip 3 in each group) or 1:1000 (strip 4 in each group). Molecular weights in kilodaltons are indicated on the right. 
sponds to WGA dimers or aggregates. Studies with the antiserum to HRP revealed two major bands: a 60 - to 64-kd band corresponding to the WGA-HRP conjugate and a 40 to $45-\mathrm{kd}$ band corresponding to free HRP (Fig. 3, C and D). A weak immunoband migrating at about $80 \mathrm{kd}$ probably represents polymeric conjugates since some faint bands of a similar molecular weight were seen also in strips stained with the lower dilutions of the anti-WGA antiserum. HRP breakdown products were only detected with low dilutions of the anti-HRP antiserum.

Neither incubation of WGA-HRP with $\mathrm{N}$-acetylglucosamine nor repeated freezing and thawing of this conjugate altered its immunoreactivity nor that of free WGA and HRP. WGA-HRP diluted in PBS and stored at $4^{\circ} \mathrm{C}$ for 2 weeks and 3 months did not behave significantly different from freshly solubilized WGA-HRP in gels and immunoblots.

Optimal dilutions of anti-WGA and anti-HRP antisera for the detection of injected WGA-HRP in tissue extracts were determined in immunoblots prepared from gels containing electrophoresed submandibular gland or superior cervical ganglion homogenates from uninjected rats to which different amounts of WGA-HRP, WGA, or HRP were added. In these experiments (some of which are illustrated in Fig. 4), the antiserum to HRP diluted 1:1000 and the antiserum to WGA diluted 1:500 gave optimal results without cross-reacting with

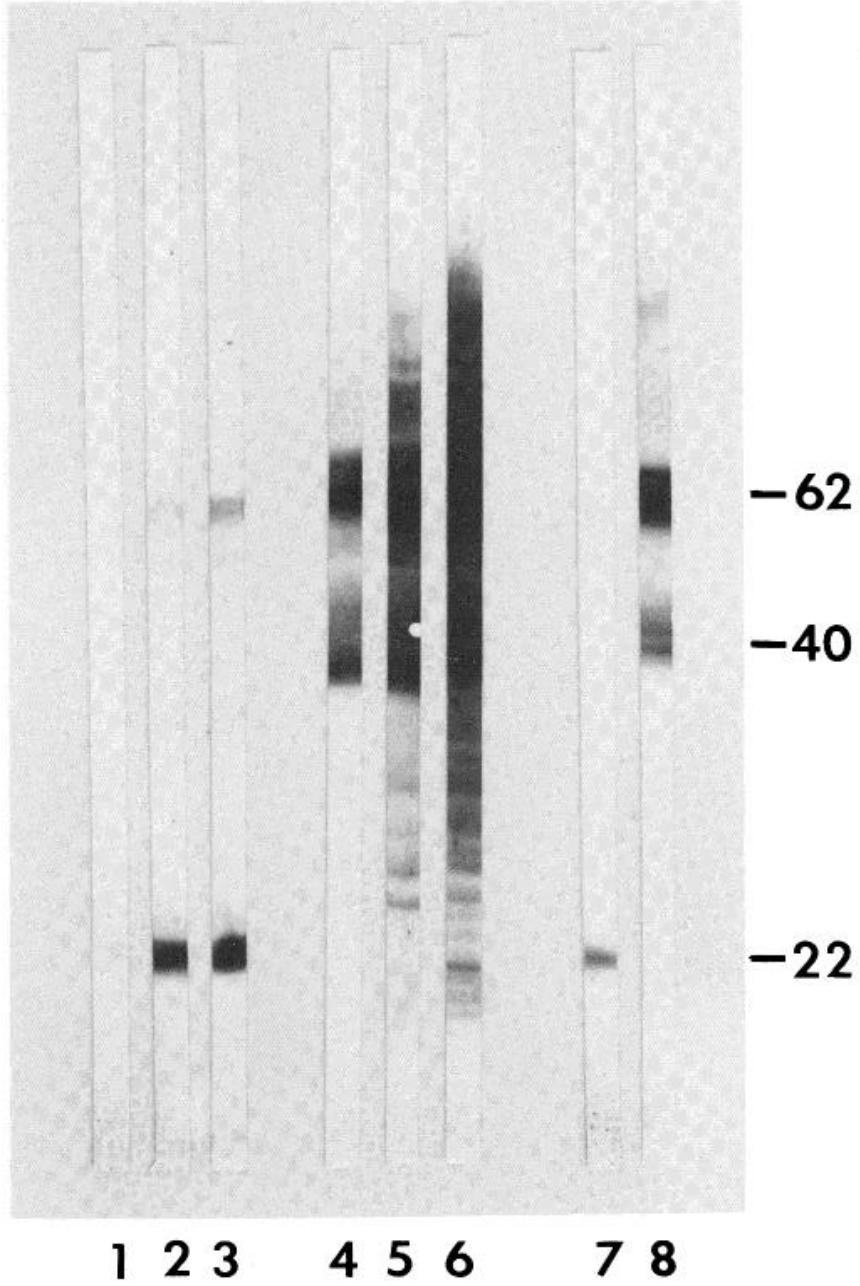

Figure 4. Strips from nitrocellulose replicas of gradient gels containing either $10 \mu \mathrm{g}$ (strips 1, 4, 7, and 8), $50 \mu \mathrm{g}$ (strips 2 and 5), or $100 \mu \mathrm{g}$ (strips 3 and 6) of WGA-HRP per $3.5-\mathrm{cm}$-wide lane. The WGA-HRP was admixed with submandibular gland (strips 1 to 6 ) or superior cervical ganglion (strips 7 and 8 ) homogenates prior to electrophoresis as described in the text. The strips were immunostained with antiserum to WGA diluted 1:500 (strips 1 to 3 and 7) or to HRP (strips 4 to 6 and 8 ) diluted 1:1000.

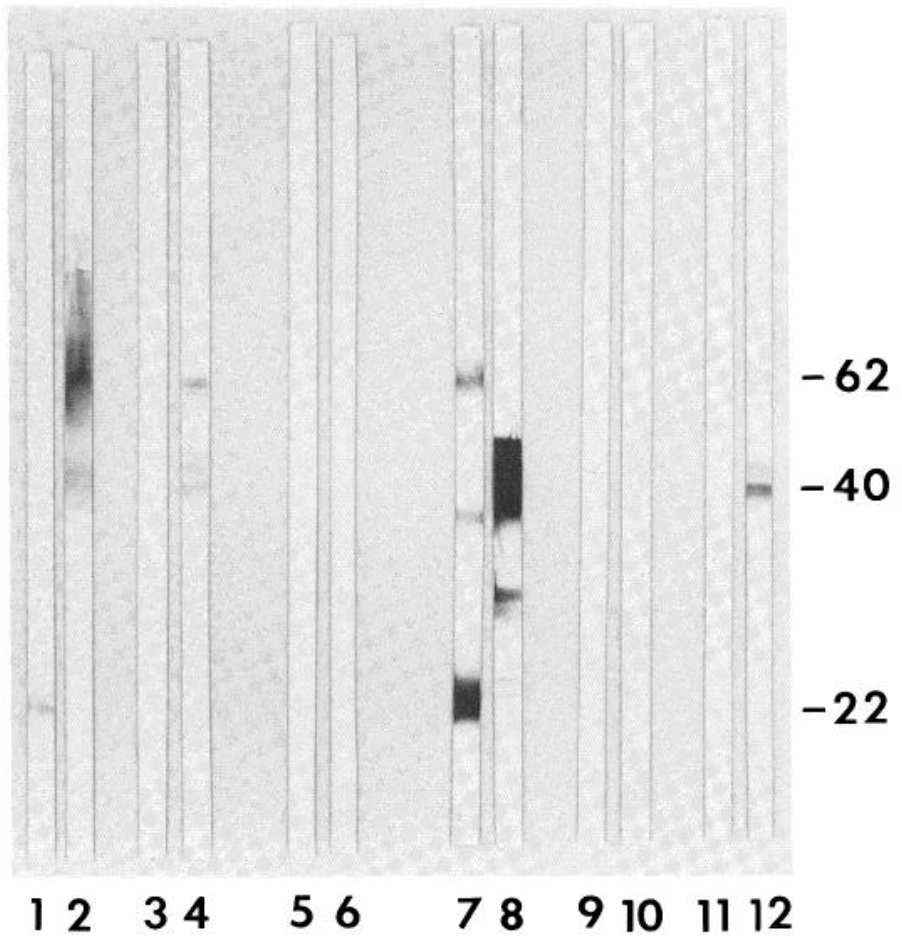

Figure 5. Nitrocellulose strips from replicas of gradient gels containing submandibular gland (strips $1,2,5,9$, and 10 ) or superior cervical ganglion (strips 3, 4, 6, 11, and 12) homogenates from rats injected with WGA-HRP (strips 1 to 4 and 9 and 10) or PBS (strips 5 and 6). The WGA-HRP rats were sacrificed 1 day (strips 1 to 4 ) or 3 days (strips 9 to 12) after the injections. Control strips (strips 7 and 8 ) are from similar gradient gels containing either WGA-HRP (strip 7) or HRP (strip 8). Each strip was immunostained with either antiserum to WGA diluted 1:500 (strips 1, 3, 7, 9, and 11) or to HRP diluted 1:500 (strips $2,4,5,6,10$, and 12). For strip 8 , anti-HRP antiserum was diluted 1:1000. Molecular weights in kilodaltons are indicated on the right.

submandibular gland or superior cervical ganglion proteins (compare Fig. 4 with Figs. 1 and 3). The admixture of these protein extracts with WGA-HRP, WGA, and HRP reduced the sensitivity of this method slightly compared to immunoblots prepared from strips containing only WGA-HRP, WGA, or HRP. Immunoblots of submandibular gland and superior cervical ganglion homogenates from PBSinjected rats confirmed that there was no cross-reaction with proteins in these tissues when the antisera to HRP and WGA were diluted $1: 500$ or more.

Immunochemical detection of WGA-HRP with anti-HRP antiserum in superior cervical ganglion and submandibular gland homogenates from injected rats. Nitrocellulose strips from the superior cervical ganglia of WGA-HRP-injected rats immunostained with antiserum to HRP detected retrogradely transported WGA-HRP as a 62-kd immunoband at survival times of $24 \mathrm{hr}, 36 \mathrm{hr}$, and 3 days (Fig. 5). The other major immunoband stained by this antiserum migrated at $40 \mathrm{kd}$ and corresponded to unconjugated HRP. At 24 $\mathrm{hr}$, the conjugate was more prominent, whereas at 3 days, free HRP was more prominent. Low molecular weight immunobands corresponding to HRP degradation products were not evident. After survival times of $16 \mathrm{hr}$ or of 5 and 8 days, no immunobands could be detected with the anit-HRP antiserum.

Immunoblots of submandibular glands prepared 24 and $36 \mathrm{hr}$ after a WGA-HRP injection showed two intensely stained immunobands at 62 and $40 \mathrm{kd}$, corresponding to the WGA-HRP conjugate and free HRP, respectively. Immunoblots from the $24 \mathrm{hr}$ group of rats are seen in Figure 5. After a 3-day survival interval, only one weakly stained band at $40 \mathrm{kd}$ was seen, and none were present after 5- and 8-day survivals. For the 36-hr experiment, both the supernatants and the pellets from the superior cervical ganglion and 
submandibular gland homogenates were analyzed. Immunoblots of the pellets did not reveal any immunobands. Thus, as is the case with free HRP (Schmidt et al., 1985), the biochemical procedures used here release conjugated and free WGA and HRP into the supernatant.

Immunochemichal detection of WGA-HRP with anti-WGA antiserum in superior cervical ganglion and submandibular gland homogenates from injected rats. Strips of electroblots adjacent to the ones used for immunostaining with anti-HRP antiserum were reacted with antiserum to WGA. This revealed a strong $22-\mathrm{kd}$ immunoband corresponding to free, monomeric WGA in the submandibular gland injected $24 \mathrm{hr}$ before processing (Fig. 5). No other immunobands were seen at this or other time points in the injected submandibular glands or in the superior cervical ganglia of injected rats at antiserum dilutions of 1:500 or higher. Lower dilutions of the antiserum yielded background staining. No better results were obtained with antiserum to WGA raised in the same or other species (M. L. Schmidt and J. Q. Trojanowski, unpublished observation).

Histochemical and histological data. At all survival times, densly labeled neurons were found in each superior cervical ganglion of the WGA-HRP-injected rats (Fig. 6). Reaction product was confined to neurons; none was seen in glial or other superior cervical ganglion cells indicative of intercellular transfer of WGA-HRP. Furthermore, there was no evidence of pathological alterations in the superior cervical ganglia of these rats, even in the the rat which received an injection of $1 \mathrm{mg}$ of WGA-HRP. Portions of the submandibular gland from this animal were examined in hematoxylin and eosin-stained paraffin sections. It appeared the same (Fig. 7) as the submandibular gland of the PBS-injected control animal.

Enzymatic activity of WGA-HRP in nondenaturing gels. As described previously (Schmidt et al., 1985), the peroxidatic activity of HRP is preserved in nondenaturing gels. When WGA-HRP was similarly evaluated, the proteins of the conjugate did not resolve as well as in the SDS gels. Reaction product was present throughout the gel in overloaded lanes (15 $\mu \mathrm{g}$ of WGA-HRP), but in all of the lanes it was concentrated at the top of the gel, suggesting that aggregated protein was not entering the gel. This was also observed with similar gels of free HRP (Schmidt et al., 1985). When less protein was loaded ( $1 \mu \mathrm{g}$ of WGA-HRP), reaction product was concentrated in the region of the conjugate and the free HRP, but diffuse reaction product was seen throughout the gel.

Discussion. A number of studies have shown that in vivo neurons

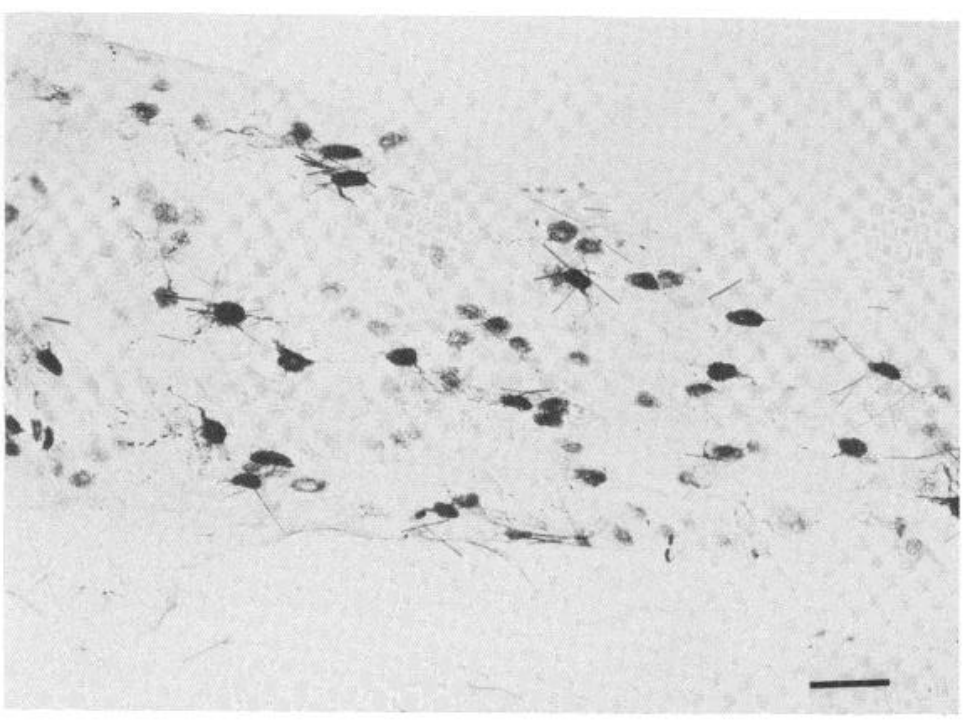

Figure 6. Photomicrograph of a section from the superior cervical ganglion of a rat sacrificed 2 weeks after an injection of $1 \mathrm{mg}$ of WGAHRP. Peroxidatic activity in the retrogradely labeled neurons was demonstrated histochemically. Bar $=100 \mu \mathrm{m}$. No counterstain.

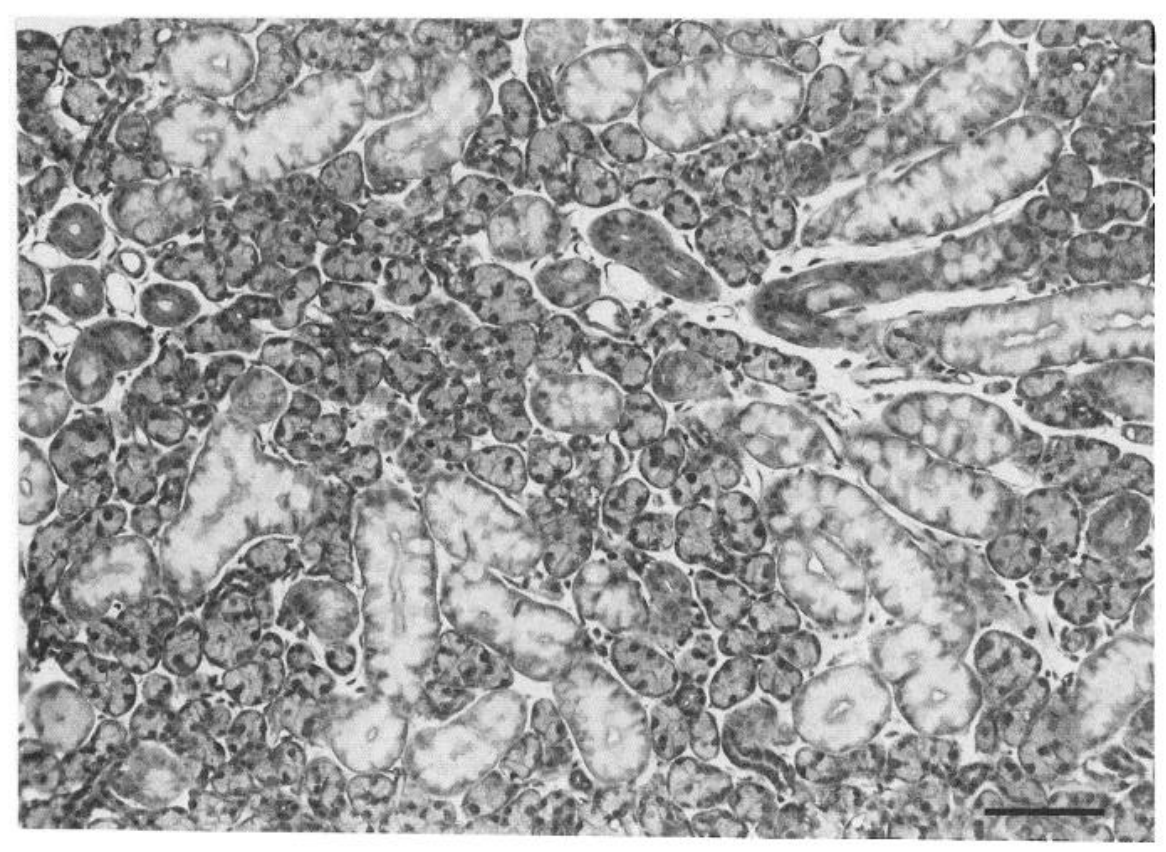

Figure 7. Photomicrograph of a section from the submandibular gland of a rat injected with $1 \mathrm{mg}$ of WGA-HRP and sacrificed 2 weeks later. In this hematoxylin and eosin-stained section, the normal morphology of the mucinous and serous portions of the submandibular gland is seen. No pathological changes are evident. $B a r=200 \mu \mathrm{m}$. 
respond to WGA-HRP differently from native HRP (for reviews see Gonatas, 1982; Trojanowski, 1983a). Nevertheless, no previous studies have directly examined the assumption that the peroxidatic activity in a neuron, following exposure of the neuron to WGA-HRP, results exclusively from the presence of HRP in a conjugated form.

Here we have characterized for the first time the proteins present in a purified WGA-HRP preparation prior to injection, at the injection site, and in retrogradely labeled neurons. Our biochemical and immunochemical data on freshly solubilized WGA-HRP preparations indicate that, although the predominant immunoband seen with antiWGA or anti-HRP antiserum is a 62-kd band corresponding to a WGA-HRP conjugate comprised of a 1:1 ratio of monomeric WGA and HRP, other immunobands are identified with these antisera. The major additional immunobands have apparent molecular weights which correspond to those of unconjugated, monomeric WGA and unconjugated HRP; minor additional immunobands correspond to dimeric WGA and spontaneous degradative products of HRP. Studies of this WGA-HRP preparation in nondenaturing gels indicate that WGA-HRP, unconjugaled HRP, and other forms of HRP have peroxidatic activity. We conclude that the peroxidase visualized in neurons exposed to WGA-HRP represents the enzymatic activity of both WGA-HRP and unconjugated HRP

These data are important for morphological studies which employ WGA-HRP or similar conjugates to delineate the complex intraneuronal pathways available for the transport of ligands. Neuronal vesicles participating in the intracellular transport of hybrid ligands such as WGA-HRP may include compartments which contain only unconjugated, enzymatically active HRP. Thus, peroxidase-containing organelles labeled after exposure of neurons to WGA-HRP may contain only WGA-HRP or unconjugated HRP or both forms of HRP. Since studies of the same populations of neurons with either WGAHRP or unconjugated HRP indicate that only those neurons exposed to WGA-HRP contain peroxidase-labeled cisterns at the trans-face of the Golgi apparatus (Harper et al., 1980; Trojanowski et al., 1981; Gonatas, 1982; Trojanowski 1983a; Trojanowski and Gonatas, 1983; Broadwell et al., 1984), it is likely that this compartment participates in the disposition of WGA-HRP but not in the transport of native HRP. However, in other cell types, such as somatotrophs of the pituitary gland, frec HRP may enter this compartment (Broadwell and Oliver, 1983).

However, other organelles, such as smooth and coated vesicles, lysosomes, tubules, multivesicular bodies, and so on, are labeled after neurons are exposed to WGA-HRP or native HRP (Schwab et al., 1979; Harper et al., 1980; Trojanowski et al., 1981; Gonatas, 1982; Trojanowski, 1983a; Trojanowski and Gonatas, 1983; Broadwell et al., 1984; LaVail and Sugino, 1984). These vesicles probably include some which contain only WGA-HRP and some which contain native HRP or both HRP and WGA-HRP. Since degradation products of HRP have peroxidatic activity (Schmidt et al., 1985), WGA-HRP preparations initially devoid of free HRP could generate enzymatically active HRP breakdown products either in solution, at the injection site, or following internalization by neurons.

Differences in the subcellular localization of internalized native WGA (Ruda and Coulter, 1982), 125-WGA (LaVail et al., 1983), and WGA-HRP (Schwab et al., 1979; Harper et al., 1980; LaVail and Sugino, 1984; Trojanowski et al., 1981; Trojanowski and Gonatas, 1983; LaVail and Sugino, 1984) in neurons have been reported. Some of these differences are probably due to differences in the derivatized forms of WGA used in these studies (Trojanowski, 1983a, b; LaVail and Sugino, 1984). The presence of unconjugated HRP in WGA-HRP preparations may also contribute to these differences.

The reasons for our inability to detect immunoreactive free or conjugated WGA in most injected submandibular glands and following retrograde transport to the superior cervical ganglia are not clear. The interaction of WGA with the hapten sugar to which it binds may alter its immunoreactivity. This interpretation is supported by the studies of WGA-HRP admixed with superior cervical ganglion or submandibular gland homogenates from uninjected rats. However, immunoblots of WGA-HRP admixed with $N$-acetylglucosamine did not appreciably inhibit the detection of WGA with this antiserum. We conclude that WGA is immunochemically altered following injection, inernalization, and transport.

The methods described here should permit insights into the mechanisms utilized by neurons to degrade and eliminate other internalized proteins. The different degradative pathways neurons employ for a variety of endogenous and exogenous proteins under both normal and pathological conditions could be delineated with these methods. Such data could be correlated with morphological observations on the different vesicles which participate in the translocation of proteins from the cell surface of a neuron to their ultimate destination. These methods could also be used to probe the dramatic rearrangements in the Golgi apparatus which occur following stress or exposure to a defined stimulus (Broadwell and Oliver, 1981; Hickey et al., 1983). Such information will lead to a better understanding of the way neurons internalize and dispose of growth factors, hormones, toxins, and other pathogens.

\section{References}

Broadwoll, R. D., and C. Oliver (1981) Golgi apparatus, GERL and secretory granule formation within neurons of the hypothalamo-hypophyseal system of control and hyperosmotically stressed mice. J. Cell Biol. 90: 474-484.

Broadwell, R. D., and C. Oliver (1983) Arr erıyrne cytochernical study of the endocytic pathways in anterior pituitary cells of the mouse in vivo. $\mathrm{J}$. Histochem. Cytochem. 31: 325-335.

Broadwell, R. D., A. M. Cataldo, and B. I. Balin (1984) Further studies of the secretory process in hypothalamo-neurohypophysial neurons: An analysis using immunocytochemistry, wheat germ agglutinin-peroxidase, and native peroxidase. J. Comp. Neurol. 228: 155-167.

Gerfen, C. R., D. D. M. O'Leary, and W. M. Cowan (1982) A note on the transneuronal transport of wheat germ agglutinin-conjugated horseradish peroxidase in the avian and rodent visual system. Exp. Brain Res. 48: 443-448.

Goldstein, I. J., and C. E. Hayes (1978) The lectins: Carbohydrate-binding proteins of plants and animals. Adv. Carbohydr. Chem. Biochem. 35: 127340.

Gonatas, N. K. (1982) The role of the neuronal Golgi apparatus in a centripetal membrane vesicular traffic. J. Neuropathol. Exp. Neurol. 41: 6-17.

Gonatas, N. K., C. Harper, T. Mizutani, and J. O. Gonatas (1979) Superior sensitivity of conjugates of horseradish peroxidase with wheat germ agglutinin for studies of retrograde axonal transport. J. Histochem. Cytochem. 27: 728-734.

Grafstein, B., and D. Forman (1980) Intracellular transport in neurons. Physiol Rev. 60: $1167-1283$

Harper, C., J. O. Gonatas, A. Stieber, and N. K. Gonatas (1980) In vivo uptake of wheat germ agglutinin-horseradish peroxidase conjugates into neuronal GERL and lysosomes. Brain Res. 188: 465-472.

Hickey, W. F., A. Stieber, R. Hogue-Angeletti, J. Gonatas, and N. K. Gonatas (1983) Nerve growth factor induced changes in the Golgi apparatus of $\mathrm{PC}-12$ rat pheochromocytoma cells as studied by ligand endocytosis cytochemical and morphometric methods. J. Neurocytol. 12: 751-756.

Itaya, S. K., and G. W. Van Hoesen (1982) WGA-HRP as a transneuronal marker in the visual pathways of monkeys and rat. Brain Res. 236: 199204

Laemmli, U. K. (1970) Cleavage of structural proteins during the assembly of bacteriophage T4. Nature 227: 680-685

LaVail, J. H., and I. K. Sugino (1984) Localization of axonally transported label in chick retinal ganglion cell axons after intravitreal injections of wheat germ agglutinin conjugated to horseradish peroxidase. Brain Res. 304. 59-69.

LaVail, J. H., I. K. Sugino, and D. M. McDonald (1983) Localization of axonally transported [125-I] wheat germ agglutinin beneath the plasma membrane of chick ganglion cells. J. Cell Biol. 96: 373-381

Lee, V. M. -Y., C. D. Page, H. -L. Wu, and W. W. Schlaepfer (1984) Monoclonal antibodies to gel-excised glial filament protein and their reactivities with other intermediate filament proteins. J. Neurochem. 42: 25-32.

Margolis, T. P., C. M. F. Marchand, H. B. Kistler, and J. H. LaVail (1981) Uptake and anterograde axonal transport of wheat germ agglutinin from retina to optic tectum in the chick. J. Cell Biol. 89: 152-156.

Nagata, Y., and M. W. Burger (1974) Wheat germ agglutinin: Molecular characteristics and specificity for sugar binding. J. Biol. Chem. 249: 31163122. 
Nygren, $\mathrm{H}$. (1982) Conjugation of horseradish peroxidase to Fab fragments with different homobifunctional and heterobifunctional cross-linking reag. .s: A comparative study. J. Histochem. Cytochem. 30: 407-412.

O'Sullivan, M .J., E. Gnemmi, D. Morris, G. Chieregatti, M. Simmons, A. D. Simmonds, J. W. Bridges, and V. Marks (1978) A simple method for the preparation of enzyme-antibody conjugates. FEBS Lett. 95: 311-313.

Raikhel, N. V., M. L. Mishkind, and B. A. Palevitz (1984) Characterization of a wheat germ agglutinin-like lectin from adult wheat plants. Planta Med. 162: $55-61$

Rice, R. H., and M. E. Etzler (1975) Chemical modification and hybridization of wheat germ agglutinins. Biochemistry 14: 4093-4099.

Ruda, M., and J. D. Coulter (1982) Axonal and transneuronal transport of wheat germ agglutinin demonstrated by immunocytochemistry. Brain Res. 249: $237-246$.

Schmidt, M. L., V. M. -Y. Lee, and J. O. Trojanowski (1985) Immunochemical detection of diverse molecular species of horseradish peroxidase recovered from rat superior cervical ganglion following retrograde transport Brain Res. 328: 65-72

Schwab, M. E., F. Javory-Agid, and Y. Agid (1978) Labeled wheat germ agglutinin (WGA) as a new highly sensitive retrograde tracer in the rat brain hippocampal system. Brain Res. 152: 145-150.

Schwab, M. E., K. Suda, and H. Thoenen (1979) Selective retrograde transsynaptic transfer of a protein, tetanus toxin, subsequent to its retrograde axonal transport. J. Cell Biol. 82: 789-810.
Steindler, D. A., and R. H. Bradley (1983) $\mathrm{N}$-[acetyl ${ }^{3} \mathrm{H}$ ] wheat germ agglutinin: Anatomical and biochemical studies of a sensitive bidirectionally transported tracer. Neuroscience 10: 219-241.

Towbin, H., T. Staehelin, and J. Gordon (1979) Electrophoretic transfer of proteins from polyacrylamide gels to nitrocellulose sheets: Procedure and some applications. Proc. Natl. Acad. Sci. U. S. A. 76: 4350-4353.

Trojanowski, J. Q. (1983a) Native and derivatized lectins for in vivo studies of neuronal connectivity and neuronal cell biology. J. Neurosci. Methods 9: $185-204$

Trojanowski, J. Q. (1983b) Time of arrival of wheat germ agglutinin-HRP conjugates in superior colliculus after intraocular injections in the rat. Brain Res. 267: 365-370.

Trojanowski, J. Q., and N. K. Gonatas (1983) A morphometric study of the endocytosis of wheat germ agglutinin-horseradish peroxidase conjugates by retinal ganglion cells in the rat. Brain Res. 272: 201-210.

Trojanowski, J. Q., and M. L. Schmidt (1984) Interneuronal transfer of axonally transported proteins: Studies with HRP and HRP conjugates of wheat germ aggiutinin, cholera toxin and the $B$ subunit of cholera toxin. Brain Res. 311:; 366-369.

Trojanowski, J. Q., J. O. Gonatas, and N. K. Gonatas (1981) A light and electron microscopic study of the intraneuronal transport of horseradish peroxidase and wheat germ agglutinin-peroxidase conjugates in the rat visual system. J. Neurocytol. 10: $441-456$ 